\title{
Spontaneous Arterial Dissection- Risk Factors And Clinical Features: Study Of 70 Cases And Review Of Literature
}

\author{
Haniyeh Javanmardi ${ }^{1}$, Saba Nouri kouchi ${ }^{1}$, Kimia Goodarzi ${ }^{1}$, M ohammad Javad Nobakht $^{1}$, \\ Fatemeh Edraki ${ }^{1}$, Reza Hosseini ${ }^{1}$, Amin Jazayeri ${ }^{1}$, Afshin Borhani Haghighi ${ }^{2 *}$ \\ ${ }^{1}$ Clinical neurology Research Center, Student Research Committee, Shiraz University of M edical Sciences, Shiraz, Iran. \\ ${ }^{2}$ Clinical Neurology Research Center, Department of Neurology, Shiraz University of Medical Sciences, Shiraz, Iran. \\ ${ }^{*}$ Corresponding Author
}

Dissection refers to a serious condition that begin as a tear or rupture in the inner layers of large vessel wall. Tear allows the blood to surges through the layers, leads to intramural hematoma or an aneurysmal dilatation, either of which can be a source of thromboembolism. Ischemic symptoms in surrounding structures are more often secondary to embolism. Dissections are labeled spontaneous in the absence of major blunt or penetrating trauma. The incidence rates for spontaneous carotid artery dissection have been reported to be 2.5 to 3 per 100,000 population. The average annual incidence for vertebral artery dissection were calculated to be 1 to 1.5 per 100,000. Typical clinical features seen with dissection include unilateral headache, ipsilateral Homer's syndrome, oculosympathetic palsy, prominent neck and facial pain, retinal ischemia, and symptoms of focal brain ischemia due to the site of dissection. The reason of dissection occurrence is still unknown but numerous factors e.g. connective tissue disorders, hyperhomocystinemia, fibromuscular dysplasia, recent infection, etc. may underlie spontaneous or minor trauma dissections. Patients with Spontaneous dissection are more susceptible to developing to stroke and its morbidity and mortality.

In this retrospective study, we evaluate the clinical features, risk factors, treatment and outcome of spontaneous dissection in 70 patients ( 31 female and 39 male), referred to Namazi hospital between September 2012 and September 2016. The diagnosis of arterial dissection was established by qualified physicians according to radiological and clinical features. Patients with traumatic dissection and a history of major or minor head and neck injury were excluded. Subjects were observed for multiple risk factors such as age dependent risk factors, family history of venous thrombosis, history of trauma, coagulopathies, infectious diseases, systemic disorders, arterio-venous malformations and autoimmune diseases. We also investigated for any history of OCP administration in female patients.

The collected data was evaluated using SPSS 16. Chi-square test were performed for the comparison of qualitative variables. And $p \varangle 0.05$ was considered as the level of significance in two-tailed tests. Statistical analysis of our data found a significant correlation between hypertension and occurrence of spontaneous arterial dissection. The findings confirm that hypertension, OCP administration and smoking can increase the risk of CVST significantly.

DOI: $10.7575 /$ aiac.abcmed.ca1.15

A I Published Date: February 2017

AC Peer-review is under responsibility of the 9th Iranian Stroke Congress.

Published by Australian International Academic Centre, Australia

This published work is open access under the CC BY license.

Available online at www.abcmed.aiac.org.au 